\title{
Clinical and Biomechanical Effects of Low-Dye Taping and Figure-8 Modification of Low-Dye Taping in Patients With Heel Pad Atrophy
}

\author{
You Hyeon Chae, MD ${ }^{1}$, Joo Sup Kim, MD, PhD ${ }^{1}$, Yeon Kang, $\mathrm{MD}^{2}$, Hyun Young Kim, MD ${ }^{1}$, Tae Im Yi, MD
}

${ }^{1}$ Department of Rehabilitation Medicine, Bundang Jesaeng General Hospital, Seongnam;
${ }^{2}$ Department of Rehabilitation Medicine, Samcheok Medical Center, Samcheok, Korea

Objective To investigate and compare the effect of low-dye taping (LDT) and figure-8 modification of LDT (MLDT) on peak plantar pressure and heel pain in patients with heel pad atrophy.

Methods There were reviewed 32 feet of 19 patients who have been diagnosed with heel pad atrophy who were enrolled in this study. The patients were diagnosed with heel pad atrophy with clinical findings, and loaded heel pad thickness measured by ultrasonography. At the first visit, patients were taught how to do LDT and MLDT. They were instructed to do daily living with barefoot, LDT and MLDT at least one time per day. Patients performed pedobarography with barefoot, LDT and MLDT within 2 weeks. The severity of heel pain was also checked with the visual analogue scale (VAS) during daily living with barefoot, LDT and MLDT.

Results VAS of hindfoot were significantly decreased after LDT and MLDT $(\mathrm{p}<0.01)$. Peak plantar pressure under hindfoot were also decreased after LDT and MLDT $(\mathrm{p}<0.01)$. The effect of MLDT in decreasing peak plantar pressure of hindfoot $(\mathrm{p}<0.01)$ and pain relief $(\mathrm{p}=0.001)$ was better than the effect of LDT.

Conclusion The LDT technique is clinically useful for pain management and reducing peak plantar pressure of hindfoot in patients with heel pad atrophy. MLDT is more effective than LDT in reducing peak plantar pressure and heel pain in patients with heel pad atrophy.

Keywords Heel, Pressure, Gait

\section{INTRODUCTION}

Low-dye taping (LDT), was first developed by Dr. Ralph Dye, and is a well-known technique commonly used to treat plantar heel pain including plantar fasciitis [1-5]. It has been reported that the anti-pronation effect of LDT is the main mechanism for reducing plantar heel pain [1,6-9]. LDT also has an effect of increasing the heel pad

Received May 19, 2017; Accepted July 20, 2017

Corresponding author: Tae Im Yi

Department of Rehabilitation Medicine, Bundang Jesaeng General Hospital, 20 Seohyeon-ro 180beon-gil, Bundang-gu, Seongnam 13590, Korea. Tel: +82-31-779-0063, Fax: +82-31-779-0635, E-mail: taeim@hanmail.net

ORCID: You Hyeon Chae (http://orcid.org/0000-0002-3403-0975); Joo Sup Kim (http://orcid.org/0000-0001-7541-1603); Yeon Kang (http://orcid. org/0000-0002-9093-1612); Hyun Young Kim (http://orcid.org/0000-0003-4920-8374); Tae Im Yi (http://orcid.org/0000-0001-6369-4677).

(c) This is an open-access article distributed under the terms of the Creative Commons Attribution Non-Commercial License (http://creativecommons.org/ licenses/by-nc/4.0) which permits unrestricted noncommercial use, distribution, and reproduction in any medium, provided the original work is properly cited. Copyright $\odot 2018$ by Korean Academy of Rehabilitation Medicine 
thickness $[6,10]$, therefore LDT can give a cushioning effect on the heel.

Heel pad atrophy is the second leading cause (14.8\%) of plantar heel pain [11] and must be differentially diagnosed as compared to plantar fasciitis. If heel pad atrophy has occurred, there is destruction of the fibrous membrane, loss of moisture content and decreased elasticity of the soft tissue that leads to reduced shock absorbing mechanism [12]. Therapeutic insole and heel cushion are usually used in patients with heel pad atrophy, and this application can reduce plantar heel pain by preserving heel pad and dispersing pressure under plantar area [13]. However, insole and heel cushion are difficult to apply indoors. For this reason, LDT is also commonly used in heel pad atrophy for its effect of increasing the heel pad thickness [10] and LDT can be easily be applied indoors.

In the previous studies, the changes of peak plantar pressure after LDT were different from study to study. Russo and Chipchase [14] reported that peak plantar pressure was increased under heel and lateral midfoot in healthy participants. In other study conducted by Lange et al. [7] for healthy people with navicular drop, there was a significant decrease of peak plantar pressure of heel and forefoot area. Saxelby et al. [1] and Van Lunen et al. [4] also reported a decreased peak plantar pressure of hindfoot after LDT was used in patients with plantar fasciitis. Conversely, some studies reported that there was no statistical change in plantar pressure of hindfoot after LDT $[6,15,16]$. However, previous studies regarding the change of plantar pressure after LDT, were usually performed and reviewed on healthy people, and plantar fasciitis patients. Because the biomechanical effect of orthosis is different between healthy and pathologic conditions, it might explain the inconsistent results of previous studies. Furthermore, to the best of our knowledge, there was no previous study about the change of plantar pressure only in heel pad atrophy patients.

To our knowledge, the applied LDT methods to subjects in previous studies were different from each other. There are various modified versions of LDT, which are composed of several common components such as transverse strip, cross strip, U-shaped strip and figure-8 strip $[17,18]$. Among those components of LDT, U-shaped strip and figure-8 strip would have the biomechanical effect affecting hindfoot area. Therefore, we investigated the effect of LDT, which consists of utilizing a U-shaped strip and transverse strip, and figure-8 modification of lowdye taping (MLDT) on heel pain and plantar pressure of hindfoot, but used only in heel pad atrophy patients.

\section{MATERIALS AND METHODS}

\section{Participants and characteristics}

From May 2015 to June 2016, patients who visited foot clinic of Bundang Jesaeng General Hospital and diagnosed with heel pad atrophy by clinical findings and ultrasonography were selected to be study participants. The clinical findings include that (1) pain exists in the center of the heel or along the edges of the heel, (2) pain is aggravated with longer duration of standing while barefoot or on a hard floor, (3) tenderness exists in the center of calcaneus, (4) the calcaneus is palpated. Ultrasonography was performed to measure the participants' heel pad thickness, using LOGIQ E9 (GE Healthcare, Milwaukee, WI, USA) equipped with linear probe (GE ML6-15, 4-15 $\mathrm{MHz}$ ). Patients who presented at least three of these clinical findings and who had a thickness of the heel pad of 3 $\mathrm{mm}$ or less in ultrasonography were diagnosed with heel pad atrophy [19]. Exclusion criteria included (1) patients with other musculoskeletal disease that can present plantar heel pain, like plantar fasciitis and (2) patients with neurovascular disease that can influence gait pattern. Participants were diagnosed with heel pad atrophy on their first visit to the hospital.

\section{Taping method}

\section{Low-dye taping $[17,18]$}

Taping was done (1) from the first metatarsal head to fifth metatarsal head transversely, (2) from the lateral side of fifth metatarsal head to medial side of first metatarsal head like U-shape, (3) two more taping were applied (2), letting them overlap about $2 / 3$, and (4) finally, the metatarsal head was taped transversely (Fig. 1A).

\section{Figure-8 modification of low-dye taping $[17,18]$}

Taping was done (1) from the first metatarsal head to fifth metatarsal head transversely, (2) from the first metatarsal head to the heel, and back to the first metatarsal head like water drop shape, (3) from the fifth metatarsal head to the heel, and again back to the fifth metatarsal head, (4) from the first metatarsal head to the fifth metatarsal head via the heel like number 8 shape, and (5) 

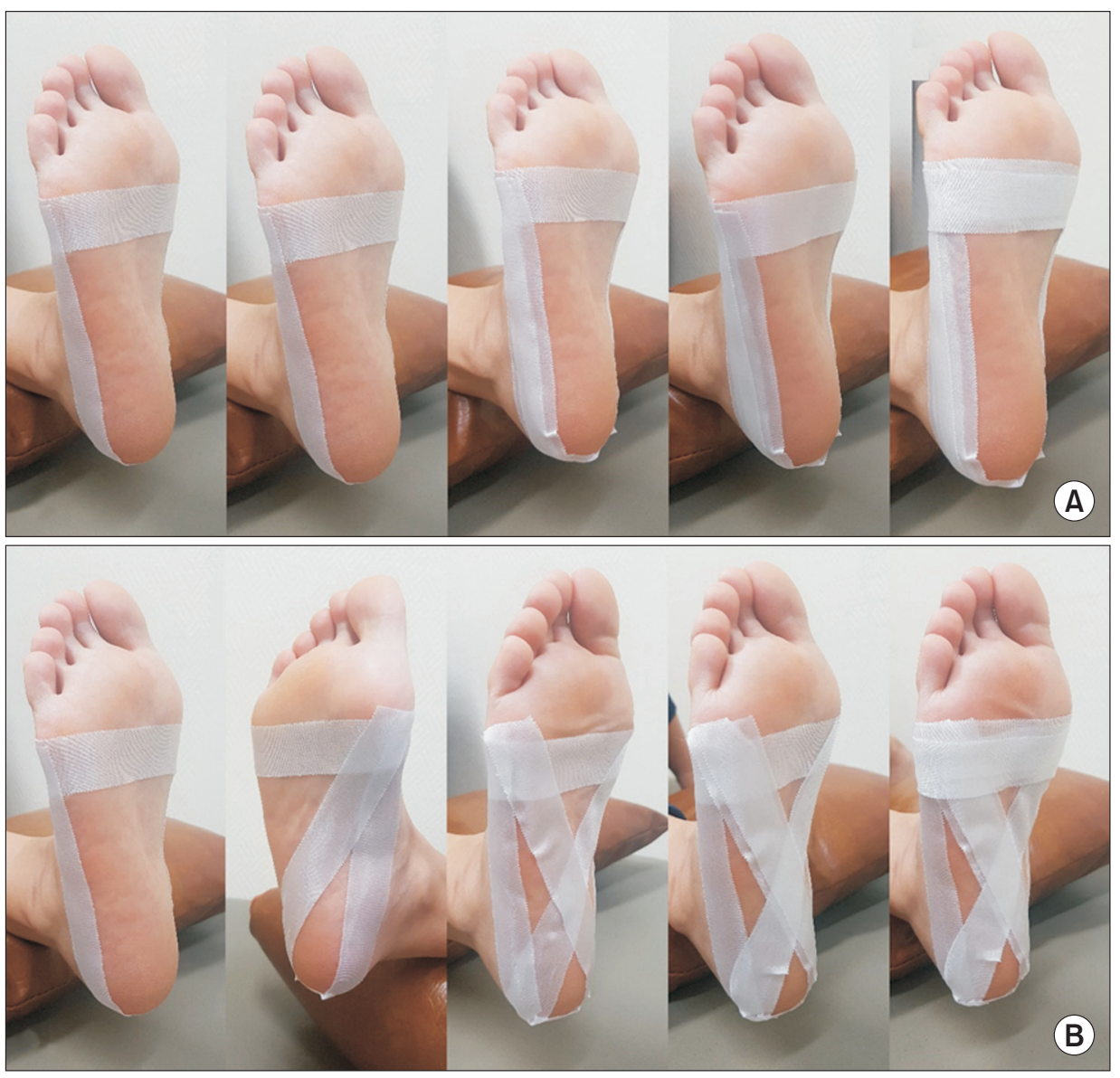

Fig. 1. (A) Low-dye taping and (B) figure-8 modification of low-dye taping. finally, the metatarsal head was transversely taped once again (Fig. 1B).

Participants learned how to do LDT and MLDT on their first visit to the hospital. We instructed participants to do their daily living with barefoot, LDT and MLDT for different timeframes each day in a week.

\section{Outcome measure}

\section{Heel pain}

Participants were instructed to record their worst heel pain during daily living with barefoot, LDT and MLDT without figure-8 strip. Plantar heel pain was recorded with visual analogue scale (VAS).

\section{Peak plantar pressure}

Participants revisited the hospital within 2 weeks. When they revisited the hospital, the researchers performed pedobargraphy via EMED system (Novel GmbH, Munich, Germany) to measure peak plantar pressure. To get rid of influence of heel pain on participant's gait pattern, pedobarography was performed only when the participant had mild plantar heel pain (less than VAS 3). Pathologic gait patterns such as limping gait or antalgic gait, were not seen during performing pedobarography. Peak plantar pressure was recorded at the random step while the participants were walking as usual, and the mean value of peak plantar pressure of five trials was used in statistical analysis. The plantar area was classified to four regions, such as toes, forefoot, midfoot and hindfoot. Peak plantar pressure was recorded from each of the studied regions. A participant performed pedobarography with barefoot, LDT and MLDT, continuously. In order to remove learning effect, each type of LDT was applied in random order after measuring with barefoot state and a participant was allowed to rest at least 5 minutes between each set of measurements.

\section{Statistical analysis}

SPSS version 18.0 for windows (SPSS Inc., Chicago, IL, USA) was used for statistical analysis. The peak plantar pressure and recorded VAS during daily living with barefoot, LDT and MLDT were compared using Wilcoxon 
signed-rank test. The relationship between the degree of change in pain $(\triangle \mathrm{VAS})$ and peak plantar pressure $(\triangle \mathrm{PPP})$ after LDT and MLDT group was analyzed using spearman correlation coefficient. Statistical results were noted as mean \pm standard deviation. The $p$-value $<0.05$ was considered statistically significant. This study was approved by the Institutional Review Board of Bundang Jesaeng General Hospital (No. RM17-04).

\section{RESULTS}

\section{Characteristic of subjects}

The total participants in the study were 19 patients (32 feet) who consisted of 5 males (9 feet) and 14 females (23 feet). Thirteen participants had heel pad atrophy in both sides. Participants were primarily middle-aged (mean, 51.5 \pm 14.1 years; range, 28-80 years), normal in BMI (mean, $22.6 \pm 2.6 \mathrm{~kg} / \mathrm{m}^{2}$; range, $17.7-28.3 \mathrm{~kg} / \mathrm{m}^{2}$ ). The mean value of loaded heel pad thickness was $1.67 \pm 0.65$ mm (Table 1).

\section{Change of heel pain during daily living after LDT and} MLDT

Table 2 shows participants' heel pain during daily living with barefoot, LDT and MLDT. Participants felt less pain after MLDT $(\mathrm{Z}=-4.81, \mathrm{p}<0.01)$ and LDT $(\mathrm{Z}=-4.82, \mathrm{p}<0.01)$ during daily living. The effect of MLDT on heel pain reduction was greater than the effect of LDT $(\mathrm{Z}=-3.345$, $\mathrm{p}=0.001$ ).

\section{Change of peak plantar pressure after LDT and MLDT}

Peak plantar pressure of hindfoot was significantly de- creased after MLDT $(\mathrm{Z}=-4.92, \mathrm{p}<0.01)$ and LDT $(\mathrm{Z}=-3.49$, $\mathrm{p}<0.01)$. Moreover, peak plantar pressure of hindfoot in MLDT group was significantly lower than that of LDT group $(\mathrm{Z}=-4.49, \mathrm{p}<0.01)$ (Table 2$)$.

\section{Relationship between $\triangle$ VAS and $\triangle$ PPP during daily living}

The degree of change in pain and peak plantar pressure was calculated. $\triangle$ VAS was defined as (VAS in barefoot VAS in each of LDT method) divided by VAS in barefoot. $\triangle \mathrm{PPP}$ was also calculated with the same method.

There was no significant relationship between $\triangle$ VAS and $\triangle \mathrm{PPP}$ after MLDT (correlation coefficient=-0.298, sig $=0.098$ ) and LDT (correlation coefficient $=-0.087$, sig=0.635) (Table 3$)$.

Table 1. Baseline characteristics of participants $(n=19)$

\begin{tabular}{lc}
\hline \multicolumn{1}{|c}{ Characteristic } & Value \\
\hline Age $(\mathrm{yr})$ & $51.5 \pm 14.1$ \\
\hline Sex & 5 \\
\hline Male & 14 \\
\hline Female & \\
Affected foot & $2(10.5)$ \\
\hline Right & $4(21.0)$ \\
\hline Left & $13(68.5)$ \\
\hline Both feet & $22.6 \pm 2.6$ \\
\hline BMI $\left(\mathrm{kg} / \mathrm{m}^{2}\right)$ & $1.67 \pm 0.65$ \\
\hline Loaded heel pad thickness $(\mathrm{mm})$ & \\
\hline
\end{tabular}

Values are presented as mean \pm standard deviation or number (\%).

BMI, body mass index.

Table 2. Change of heel pain and peak plantar pressure after low-dye taping

\begin{tabular}{lcccc}
\hline & Barefoot & MLDT & LDT & Significance \\
\hline Heel pain (VAS) & $6.5 \pm 1.7$ & $3.5 \pm 1.3$ & $4.2 \pm 1.1$ & ${ }^{\mathrm{a}, \mathrm{b}, \mathrm{c})}$ \\
\hline PPP $\left(\mathrm{N} / \mathrm{cm}^{2}\right)$ & & & & \\
\hline Toe & $38.8 \pm 18.5$ & $41.8 \pm 20.6$ & $40.5 \pm 19.6$ & b) \\
Forefoot & $49.5 \pm 19.9$ & $51.6 \pm 20.3$ & $47.9 \pm 17.1$ & \\
Midfoot & $12.0 \pm 4.2$ & $13.1 \pm 3.3$ & $12.8 \pm 5.2$ & b) \\
Hindfoot & $29.3 \pm 11.9$ & $23.2 \pm 7.0$ & $26.3 \pm 8.8$ & a,b,c) \\
\hline
\end{tabular}

Values are presented as mean \pm standard deviation.

MLDT, figure-8 modification of low-dye taping; LDT, low-dye taping; VAS, visual analogue scale; PPP, peak plantar pressure.

a) $\mathrm{p}<0.05$, indicating difference between MLDT and LDT group was statistically significant.

${ }^{b)} \mathrm{p}<0.05$, indicating difference between barefoot and MLDT group was statistically significant.

${ }^{c} \mathrm{p}<0.05$, indicating difference between barefoot and LDT group was statistically significant. 
Table 3. Relationship between $\triangle \mathrm{VAS}$ and $\triangle \mathrm{PPP}$

\begin{tabular}{lcccc}
\hline & \multirow{2}{*}{$\Delta$ VAS } & \multirow{2}{*}{$\Delta \mathbf{P P P}$} & \multicolumn{2}{c}{ Correlation between $\Delta$ VAS and $\Delta$ PPP } \\
\cline { 4 - 5 } & & & Coefficient & p-value \\
\hline After MLDT & $-0.5 \pm 0.2$ & $-0.2 \pm 0.1$ & -0.298 & 0.098 \\
\hline After LDT & $-0.3 \pm 0.2$ & $-0.1 \pm 0.1$ & -0.087 & 0.635 \\
\hline
\end{tabular}

Values are presented as mean \pm standard deviation.

VAS, visual analogue scale; PPP, peak plantar pressure; MLDT, figure-8 modification of low-dye taping; LDT, low-dye taping.

\section{DISCUSSION}

The results of our study demonstrate that LDT and MLDT reduce peak plantar pressure of hindfoot and plantar heel pain during daily living in heel pad atrophy patients.

Previous studies have shown the effect of LDT in patients with plantar heel pain. According to the previous studies conducted by Radford et al. [2], LDT group reported $31 \mathrm{~mm}$ reduction in first step pain (71.4 to 41.4) on $100 \mathrm{~mm}$ VAS and this effect was superior than sham group (72.0 to 54.0) in plantar fasciitis patients. Landorf et al. [5] and Ha et al. [6] also reported reduced heel pain after LDT. Our study, for patients with heel pad atrophy, showed reduced plantar heel pain after MLDT (6.5 to 3.5) and LDT (6.5 to 4.2), so LDT is also proven to be effective in patients with heel pad atrophy. LDT is often thought as a temporary brace because of loosening of taping over time $[15,20]$. Therefore, our study has the strength of recording significant reduction of the worst pain 'during daily living,' while previous studies recorded immediate pain relief after LDT. Our result suggests that LDT can be clinically feasible in daily living.

We hypothesized that peak plantar pressure would be decreased after LDT because of heel cushioning effect. In our study, there was significant decrease of peak plantar pressure under hindfoot area after applying LDT and MLDT, which is consistent with previous studies conducted by Lange et al. [7], Saxelby et al. [1], and Van Lunen et al. [4]. For accurate measurement of the changes in peak plantar pressure, we tried to eliminate factors that could affect plantar pressure during gait. First, we only included patients with heel pad atrophy in this study. Each disease that can cause plantar heel pain has a different pathology and the response to LDT can vary from disease to disease. Second, previous studies did not describe about participant's heel pain during measur- ing pedobarography. Plantar heel pain during gait could influence participant's gait pattern and also influence recorded peak plantar pressure during gait. Therefore, we measured peak plantar pressure only when the participant's heel pain was less than VAS 3. Gait speed also can influence plantar pressure of heel. We instructed participants to perform pedobarography with usual walking speed. In our study, gait speed during pedobarography were not different with barefoot $(0.29 \pm 0.05 \mathrm{~m} / \mathrm{s})$, LDT $(0.29 \pm 0.06 \mathrm{~m} / \mathrm{s})$, and MLDT $(0.28 \pm 0.05 \mathrm{~m} / \mathrm{s})$.

In our study, heel pain and peak plantar pressure of hindfoot were reduced after MLDT and LDT. Even though there was no statistically significant relationship between $\triangle \mathrm{VAS}$ and $\triangle \mathrm{PPP}$, there was tendency that patient with greater reduction in heel pain also had more change in peak plantar pressure of hindfoot. This result might support our hypothesis that LDT reduces heel pain by reducing peak plantar pressure of hindfoot.

LDT technique usually restricts excessive pronation of subtalar joint and mid-tarsal joint [4-6,9,17]. LDT also has a similar effect to heel cushion. Ha et al. [6] reported that loaded heel pad thickness was increased after LDT, and Yi et al. [10] also reported that heel pad thickness and compressibility of heel pad increased after LDT. LDT has a variety of modified versions, and one of the most commonly used modifications is adding figure-8 taping around the heel area $[16,17]$. Ator et al. [21] compared the changes in navicular height when LDT and figure-8 modified version of LDT were performed, but there was no statistical difference in the change of navicular height according to different LDT methods. There was no previous studies comparing the change of peak plantar pressure with different LDT methods. In this study, MLDT was more effective in reducing heel pain and the peak plantar pressure of hindfoot than LDT. Figure-8 and water drop shape taping around hindfoot would play a role in gathering the soft tissue of the hindfoot and a role similar to 
the plantar fascia, which is supporting longitudinal arch of foot.

This study has some noted limitations. First, the number of participants was small. Second, we did not measure the change of heel pad thickness after LDT, therefore we were not able to check results regarding the relationship between the change of heel pad thickness and the change of peak plantar pressure. Third, because we only separate plantar area into four pieces, we could not check the effect of LDT about restricting excessive pronation. Lastly, we did not ask participants about their preference for each of the application taping methods. With that information, our results would be more helpful to choose specific LDT method for management in patients with heel pad atrophy.

In conclusion, both type of LDT are clinically effective for heel pain reduction in patients with heel pad atrophy and reduce peak plantar pressure under hindfoot like heel cushion. Also, MLDT is more effective taping technique for heel pain management than LDT in patients with heel pad atrophy. Further studies are needed to determine whether MLDT is more effective than LDT in patients with other plantar heel pain, such as plantar fasciitis.

\section{CONFLICT OF INTEREST}

No potential conflict of interest relevant to this article was reported.

\section{REFERENCES}

1. Saxelby J, Betts RP, Bygrave CJ. 'Low-dye' taping on the foot in the management of plantar fasciitis. Foot (Edinb) 1997;7:205-9.

2. Radford JA, Landorf KB, Buchbinder R, Cook C. Effectiveness of low-dye taping for the short-term treatment of plantar heel pain: a randomised trial. BMC Musculoskelet Disord 2006;7:64.

3. Park C, Lee S, Lim DY, Yi CW, Kim JH, Jeon C. Effects of the application of Low-Dye taping on the pain and stability of patients with plantar fasciitis. J Phys Ther Sci 2015;27:2491-3.

4. Van Lunen B, Cortes N, Andrus T, Walker M, Pasquale $\mathrm{M}$, Onate J. Immediate effects of a heel-pain orthosis and an augmented low-dye taping on plantar pres- sures and pain in subjects with plantar fasciitis. Clin J Sport Med 2011;21:474-9.

5. Landorf KB, Keenan AM, Herbert RD. Effectiveness of foot orthoses to treat plantar fasciitis: a randomized trial. Arch Intern Med 2006;166:1305-10.

6. Ha HS, Hwang JH, Kim YB, Ha YM, Hwang JM. Immediate clinical and biomechanical effects of lowdye taping in patients with plantar heel pain. Korean J Sports Med 2012;30:9-15.

7. Lange B, Chipchase L, Evans A. The effect of low-dye taping on plantar pressures, during gait, in subjects with navicular drop exceeding $10 \mathrm{~mm}$. J Orthop Sports Phys Ther 2004;34:201-9.

8. Vicenzino B, McPoil T, Buckland S. Plantar foot pressures after the augmented low dye taping technique. J Athl Train 2007;42:374-80.

9. O'Sullivan K, Kennedy N, O'Neill E, Ni Mhainin U. The effect of low-dye taping on rearfoot motion and plantar pressure during the stance phase of gait. BMC Musculoskelet Disord 2008;9:111.

10. Yi TI, Shim JW, Ku HK, Seo IS, Huh WS, Kim JS, et al. The effects of modified low-dye taping in the patient with heel pad atrophy. J Korean Acad Rehabil Med 2011;35:96-100.

11. Yi TI, Lee GE, Seo IS, Huh WS, Yoon TH, Kim BR. Clinical characteristics of the causes of plantar heel pain. Ann Rehabil Med 2011;35:507-13.

12. Turgut A, Gokturk E, Kose N, Seber S, Hazer B, Gunal I. The relationship of heel pad elasticity and plantar heel pain. Clin Orthop Relat Res 1999;(360):191-6.

13. Pfeffer G, Bacchetti P, Deland J, Lewis A, Anderson $\mathrm{R}$, Davis W, et al. Comparison of custom and prefabricated orthoses in the initial treatment of proximal plantar fasciitis. Foot Ankle Int 1999;20:214-21.

14. Russo SJ, Chipchase LS. The effect of low-dye taping on peak plantar pressures of normal feet during gait. Aust J Physiother 2001;47:239-44.

15. Nolan D, Kennedy N. Effects of low-dye taping on plantar pressure pre and post exercise: an exploratory study. BMC Musculoskelet Disord 2009;10:40.

16. Newell T, Simon J, Docherty CL. Arch-taping techniques for altering navicular height and plantar pressures during activity. J Athl Train 2015;50:825-32.

17. Radford JA, Burns J, Buchbinder R, Landorf KB, Cook C. The effect of low-dye taping on kinematic, kinetic, and electromyographic variables: a systematic review. 
J Orthop Sports Phys Ther 2006;36:232-41.

18. SMCBC Curriculum Committee. Manual of athletic taping. Philadelphia: F. A. Davis; 1995. p. 26-30.

19. Rhee WI, Kim JM. Ultrasonographic findings of thickness and compressibility of heel pads in outpatient. J Korean Soc Med Ultrasound 2004;23:213-8.

20. Del Rossi G, Fiolkowski P, Morodyski MB, Bishop M,
Trimble M. For how long do temporary techniques maintain the height of the longitudinal arch? Phys Ther Sport 2004;5:84-9.

21. Ator R, Gunn K, McPoil TG, Knecht HG. The effect of adhesive strapping on medial longitudinal arch support before and after exercise. J Orthop Sports Phys Ther 1991;14:18-23. 